\title{
Functional Annotation of circRNAs in Tea Leaves After Infection by the Tea Leaf Spot Pathogen, Lasiodiplodia theobromae
}

\author{
Yuanyou Yang, ${ }^{1}$ Xinyue Jiang, ${ }^{1}$ Jiayan Shi, ${ }^{1}$ Yu Wang, ${ }^{1,2}$ Honglin Huang, ${ }^{1}$ Yuqin Yang, ${ }^{1,3}$ \\ Dongxue Li, ${ }^{1}$ Silong Jiang, ${ }^{1,2}$ Delu Wang, ${ }^{4, \dagger}$ and Zhuo Chen ${ }^{1, \dagger}$ \\ ${ }^{1}$ Key Laboratory of Green Pesticide and Agricultural Bioengineering, Ministry of Education, Guizhou \\ University, Guiyang, Guizhou 550025, China \\ ${ }^{2}$ Agricultural College, Guizhou University, Guiyang, Guizhou 550025, China \\ ${ }^{3}$ College of Tea Science, Guizhou University, Guiyang, Guizhou 550025, China \\ ${ }^{4}$ College of Forestry, Guizhou University, Guiyang, Guizhou 550025, China
}

\begin{abstract}
Tea leaf spot, caused by Lasiodiplodia theobromae, is an important disease that can seriously decrease the production and quality of tea (Camellia sinensis (L.) O. Kuntze) leaves. The analysis of circular RNA (circRNA) in tea leaves after infection by the pathogen could improve understanding about the mechanism of host-pathogen interactions. In this study, high-performance sequencing of circRNA from $C$. sinensis Fuding-dabaicha leaves that had been infected with $L$. theobromae was conducted using the Illumina HiSeq 4000 platform. In total, 192 and 153 differentially expressed circRNAs from tea leaves were significantly up- and downregulated, respectively, after infection with $L$. theobromae. A gene ontology analysis indicated that the differentially expressed circRNA-hosting genes for DNA binding were significantly enriched. The genes with significantly differential expressions that were annotated in the specified database ( $S$ genes) were $\sigma$ factor $E$ isoform 1, triacylglycerol lipase SDP1, DNA-directed RNA polymerase III subunit 2, WRKY transcription factor WRKY24, and regulator of nonsense transcripts 1 homolog. A Kyoto Encyclopedia of Genes and Genomes analysis indicated that the significantly enriched circRNA-hosting genes involved in the plant-pathogen interaction pathway were Calmodulin-domain protein kinase 5 isoform 1, probable WRKY transcription factor 33, U-box domain-containing protein 35, probable inactive receptor-like protein kinase At3g56050, WRKY transcription factor WRKY24, mitogen-activated protein kinase kinase kinase YODA, SGT1, and protein $D G S 1$. Functional annotation of circRNAs in tea leaves infected by $L$. theobromae will provide a valuable resource for future research on host-pathogen interactions.
\end{abstract}

\section{Resource Announcement}

The genus-rich fungal family Botryosphaeriaceae (phylum Ascomycota), which consists of saprobes, endophytes, and phytopathogens, mainly infects woody plants (Chethana et al. 2016; Morales-Cruz et al. 2015; Paolinelli-Alfonso et al. 2016; Phillips et al. 2013; Yan et al. 2013). Lasiodiplodia Ellis \& Everh., a clade of 25 species in this family, is highly pathogenic toward many plant species and can cause disease (Phillips et al. 2013; Yan et al. 2013). Among members of this genus, Lasiodiplodia theobromae (Pat.) Griffon \& Maubl. can cause diseases in many plant species such as canker of grapevine and olive (Pérez et al. 2018; Rodríguez-Gálvez et al. 2015); grapevine, cashew, and strawberry dieback (Cardoso et al.

\footnotetext{
${ }^{\dagger}$ Corresponding authors: Z. Chen; gychenzhuo@aliyun.com, and D. L. Wang; dlwang@gzu.edu.cn

*The $\boldsymbol{e}$-Xtra logo stands for "electronic extra" and indicates that supplementary tables are published online.
}

The author(s) declare no conflict of interest.

Accepted for publication 8 June 2021.

() 2022 The American Phytopathological Society 
2002; Rodríguez-Gálvez et al. 2015; Yildiz et al. 2014); fruit spot of eggplant, pomelo, peanut, and longan (Luo et al. 2011; Phipps and Porter 1998; Serrato-Diaz et al. 2014; Woodward et al. 2005); stem-end rot of citrus (Zheng et al. 2021); postharvest fruit rot in avocado (Garibaldi et al. 2012); gummosis of mango trees (Li et al. 2013; Serrato-Diaz et al. 2013); inflorescence blight of mango trees and longan trees ( $\mathrm{Li}$ et al. 2013; Serrato-Diaz et al. 2013, 2014); and leaf spot of tea (Li et al. 2019; Ren et al. 2019; Zhu et al. 2014). It is also known that Botryosphaeria rhodina (Berk. \& M. A. Curtis) Arx is an anamorph of $L$. theobromae. Tea leaf spot caused by $L$. theobromae is an important disease that leads to large reductions in tea yields ( $\mathrm{Li}$ et al. 2019).

It is important to study the pathogenic and resistance mechanisms in the tea host because they could influence control measures against the disease. In a previous study, messenger RNAs (mRNAs) and microRNAs (miRNAs) from $L$. theobromae and the tea plant host after leaf infection were sequenced, and the differentially expressed genes in the pathogen and plant host were used to study the pathogenic and resistance mechanisms (Jiang et al. in press). The target genes of the miRNAs against the pathogen and plant host have also been predicted (Jiang et al. in press). To improve control of the disease, fungicides with enhanced control effects against tea leaf spot caused by $L$. theobromae were screened and their action mode was studied (Bao et al. in press). Circular RNAs (circRNAs) have various biological functions and are associated with sponging microRNAs. They seem to be involved in interactions between the fungal pathogen and the plant host (Fan et al. 2020; Panda 2018). However, there is currently a lack of information about the circRNAs associated with $L$. theobromae and the plant host.

In this study, Camellia sinensis Fuding-dabaicha was grown in the greenhouse along with 3-year-old tea plants. The third or fourth leaves on each healthy twig were selected and both sides of the main vein on the leaves were punctured with four holes using sterilized syringe needles. The holes were approximately $6 \mathrm{~mm}$ apart. The inoculation sites were inoculated with conidial suspensions of $L$. theobromae strain GZHS-2017-010 $\left(10^{6}\right.$ conidia/ml, 20 to $30 \mu \mathrm{l}$ per inoculation aliquot). In the uninfected treatment (control), sterile water was used to inoculate the tea leaves. There were 15 healthy twigs in the inoculated treatment and the control and the treatments were replicated three times. The infected leaves were harvested at $48 \mathrm{~h}$ postinoculation after a lesion had formed on the inoculated tea leaf.

Total RNA was isolated and purified using the TRIzol reagent (Invitrogen, Carlsbad, CA, U.S.A.) according to the manufacturer's instructions. The RNA amount and purity of each sample was quantified using NanoDrop ND-1000 (NanoDrop, Wilmington, DE, U.S.A.). The RNA integrity (RIN) was assessed using Agilent 2100 bioanalyzer (Agilent Technologies, Palo Alto, Calif. U.S.A.) with a RIN number > 7.0. Approximately $5 \mu \mathrm{g}$ of total RNA was used to deplete the ribosomal RNA according to the Ribo-Zero rRNA Removal Kit instructions (Illumina, San Diego, CA, U.S.A.). After removing the ribosomal RNAs, the remaining RNAs were fragmented into small pieces using divalent cations at high temperature. Then, the cleaved RNA fragments were reverse-transcribed to create the cDNA, which was then used to synthesize U-labeled second-stranded DNAs with Escherichia coli DNA polymerase I, RNase $\mathrm{H}$, and dUTP. An A-base was then added to the blunt ends of each strand so that they could be ligated to the indexed adapters. Each adapter contained a T-base overhang for ligating the adapter to the A-tailed fragmented DNA. Single- or dual-index adapters were ligated to the fragments and size selection was performed using AMPureXP beads (Beckman Part Number A63881; Indianapolis, IN, U.S.A.). After heat-labile UDG enzyme treatment of the U-labeled second-stranded DNAs, the ligated products are amplified using the PCR process under the following conditions: initial denaturation at $95^{\circ} \mathrm{C}$ for $3 \mathrm{~min}$; 8 cycles of denaturation at $98^{\circ} \mathrm{C}$ for $15 \mathrm{~s}$, annealing at $60^{\circ} \mathrm{C}$ for $15 \mathrm{~s}$, and extension at $72^{\circ} \mathrm{C}$ for $30 \mathrm{~s}$; and a final extension at $72^{\circ} \mathrm{C}$ for 5 min. The average insert size for the final cDNA library was $300 \mathrm{bp}( \pm 50 \mathrm{bp}$ ). Finally, pairedend sequencing was performed on an Illumina HiSeq 4000 (LC Bio, Hangzhou, China) according to the manufacturer's instructions.

In the circRNA analysis, Cutadapt was used to remove the reads that contained contaminated adaptors, and low-quality and undetermined bases (Martin 2011). Then, sequence quality was verified using FastQC (https://www.bioinformatics.babraham.ac.uk/projects/fastqc/). Bowtie2 and TopHat2 software were used to map reads to the genome of the species (Kim et al. 2013; Langmead and Salzberg 2012). The remaining reads (unmapped reads) were mapped to genomes using TopHat-fusion software (Kim and Salzberg 2011). CIRCExplorer software was used to 
Table 1. Circular RNA (CircRNA) sequences from tea (Camellia sinensis) leaves after infection by the fungal pathogen Lasiodiplodia theobromae

\begin{tabular}{lll} 
& & \multicolumn{1}{c}{ Sample source $^{\mathbf{a}}$} \\
\cline { 2 - 3 } Sequence & Uninfected treatment & \multicolumn{1}{c}{ Infected treatment } \\
Raw reads & $141,647,262$ & $145,772,279$ \\
Valid reads & $139,330,285$ & $143,076,549$ \\
Valid bases & $20.90 \mathrm{G}$ & $21.46 \mathrm{G}$ \\
All CircRNAs & Upregulation & 192 (infected treatment versus uninfected treatment) \\
& Downregulation & 153 (infected treatment versus uninfected treatment) \\
Type 1: circRNAs & Upregulation & 192 (infected treatment versus uninfected treatment) \\
& Downregulation & 150 (infected treatment versus uninfected treatment) \\
Type 2: ciRNAs & Upregulation & 0 (infected treatment versus uninfected treatment) \\
& Downregulation & 3 (infected treatment versus uninfected treatment) \\
\hline
\end{tabular}

\footnotetext{
a The uninfected control and the infected tea leaves were punctured with four holes using sterilized syringe needles. The holes were approximately $6 \mathrm{~mm}$ apart. Then, sterile water (uninfected treatment) or the conidial suspension (infected treatment) was used to inoculate the tea leaves.

${ }^{b}$ ciRNA = intronic circRNA.
}

denovo assemble the mapped reads into circRNAs (Zhang et al. 2014, 2016) and all samples generated unique circRNAs. $R$ package-edgeR software was used to select the differentially expressed circRNAs using the following criteria: $\log _{2}$ (fold-change) $>1$ or $\log _{2}$ (fold change) $<-1$ and with a statistical significance of $P$ value $<0.05$ using Poisson distribution-based analysis (Robinson et al. 2010).

Infection by $L$. theobromae significantly up- and downregulated 192 and 153 circRNAs, respectively, in the tea leaf. In total, 342 circRNAs and 3 intronic circRNAs in the tea leaves were differentially expressed when infected with the pathogen. A gene ontology database annotation of the differentially expressed circRNA-hosting genes showed that the total number of genes (TB gene numbers) and the number of genes with significantly different expressions between infected and noninfected treatments (TS gene numbers) were 1,183 and 190, respectively. At the level of molecular function, the differentially expressed circRNA-hosting genes, which were $\sigma$ factor $E$ isoform 1, triacylglycerol lipase SDP1, DNA-directed RNA polymerase III subunit 2, WRKY transcription factor WRKY24, and regulator of nonsense transcripts 1 homolog, generally showed enriched DNA binding. The Kyoto Encyclopedia of Genes and Genomes database annotation of the differentially expressed circRNA-hosting genes identified 1,135 TB genes and 196 TS genes. The differentially expressed circRNA-hosting genes for Calmodulin-domain protein kinase 5 isoform 1, probable WRKY transcription factor 33, U-box domain-containing protein 35, probable inactive receptor-like protein kinase At3g56050, WRKY transcription factor WRKY24, mitogen-activated protein kinase kinase kinase YODA, $S G T 1$, and protein DGS1 were enriched in the plant-pathogen interaction pathway (Table 1).

The circRNAs from tea leaves infected with $L$. theobromae and uninfected leaves were sequenced and the differential expressions of the circRNAs and their hosting genes were analyzed. This dataset will provide an important resource that can be used to study the mechanism underlying the interaction between $L$. theobromae and the tea plant. The sequences of the circRNAs after the plant was infected with $L$. theobromae have been deposited in GenBank under Sequence Read Archive accession PRJNA689677. A bioinformatics analysis of circRNAs is presented in Supplementary Table S1. L. theobromae strain GZHS-2017-010 has been deposited in the China General Microbiological Culture Collection Center with the accession number CGMCC3.20151.

\section{Literature Cited}

Bao, X.-T., Yang, R., Jiang, S.-L., Zhao, J.-P., Wang, D.-L., Li, D.-X., Wu, X., Song, B.-A., and Chen, Z. A novel sulfone derivative controls Lasiodiplodia theobromae proliferation in tea leaf spot by reducing the ergosterol content. Mol. PlantMicrobe Interact. In press.

Cardoso, J. E., Vidal, J. C., Santos, A. A., Freire, F. C. O., and Viana, F. M. P. 2002. First report of black branch dieback of cashew caused by Lasiodiplodia theobromae in Brazil. Plant Dis. 86:558.
Chethana, K. W. T., Li, X. H., Zhang, W., Hyde, K. D., and Yan, J. Y. 2016. Trail decryption of molecular research on Botryosphaeriaceae in woody plants. Phytopathol. Mediterr. 55:147-171.

Fan, J., Quan, W., Li, G.-B., Hu, X.-H., Wang, Q., Wang, H., Li, X.-P., Luo, X., Feng, Q., Hu, Z. J., Feng, H., Pu, M., Zhao, J.-Q., Huang, Y.-Y., Li, Y., Zhang, Y., and Wang, W.-M. 2020. circRNAs are involved in the rice-Magnaporthe oryzae interaction. Plant Physiol. 182: 272-286. 
Garibaldi, A., Bertetti, D., Amatulli, M. T., Cardinale, J., and Gullino, M. L. 2012. First report of postharvest fruit rot in avocado (Persea americana) caused by Lasiodiplodia theobromae in Italy. Plant Dis. 96:460.

Jiang, S.-L., Yin, Q.-X., Li, D.-X., Wu, X., Wang, Y., Wang, D.-L., and Chen, Z. Integrated mRNA and small RNA sequencing for analyzing tea leaf spot pathogen Lasiodiplodia theobromae, under in vitro conditions and the course of infection. Phytopathology In press.

Kim, D., Pertea, G., Trapnell, C., Pimentel, H., Kelley, R., and Salzberg, S. L. 2013. TopHat2: Accurate alignment of transcriptomes in the presence of insertions, deletions and gene fusions. Genome Biol. 14:R36.

Kim, D., and Salzberg, S. L. 2011. TopHat-fusion: An algorithm for discovery of novel fusion transcripts. Genome Biol. 12:R72.

Langmead, B., and Salzberg, S. L. 2012. Fast gapped-read alignment with Bowtie 2. Nat. Methods 9:357-359.

Li, D.-X., Bao, X.-T., Ren, Y.-F., Wang, Y., Song, B.-A., and Chen, Z. 2019. First report of Lasiodiplodia theobromae causing leaf spot on tea plant in Guizhou Province of China. Plant Dis. 103:374.

Li, Q.-L., Guo, T.-X., Pan, Z.-B., Huang, S.-P., Mo, J.-Y., Ning, P., and Hsiang, T. 2013. An outbreak of gummosis of mango trees caused by Lasiodiplodia theobromae in Guangxi, South China. Plant Dis. 97:690.

Luo, M., Dong, Z.-Y., Bin, S.-Y., and Lin, J.-T. 2011. First report of fruit rot disease on pomelo caused by Lasiodiplodia theobromae in China. Plant Dis. 95:1190.

Martin, M. 2011. Cutadapt removes adapter sequences from high-throughput sequencing reads. EMBnet. J. 17:10-12.

Morales-Cruz, A., Amrine, K. C. H., Blanco-Ulate, B., Lawrence, D. P., Travadon, R., Rolshausen, P. E., Baumgartner, K., and Cantu, D. 2015. Distinctive expansion of gene families associated with plant cell wall degradation, secondary metabolism, and nutrient uptake in the genomes of grapevine trunk pathogens. BMC Genomics 16:469.

Panda, A. C. 2018. Circular RNAs act as miRNA sponges. Adv. Exp. Med. Biol. 1087:67-79.

Paolinelli-Alfonso, M., Villalobos-Escobedo, J. M., Rolshausen, P., Herrera-Estrella, A., Galindo, C., and López-Hernández, J. F. 2016. Global transcriptional analysis suggests Lasiodiplodia theobromae pathogenicity factors involved in modulation of grapevine defensive response. BMC Genomics 17:615.

Pérez, B. A., Niz, J., Salvador, R., Salvador, R., Berretta, M., Roca, M. E. M., Pesce, M. V., Matías, A. C., González De Urreta, M., Paccioretti, M., and Otero, M. L. 2018. First report of Lasiodiplodia theobromae causing branch canker on 'Manzanilla' olive in Northwestern Argentina. Plant Dis. 102:677.

Phillips, A. J. L., Alves, A., Abdollahzadeh, J., Slippers, B., Wingfield, M. J., Groenewald, J. Z., and Crous, P. W. 2013. The Botryosphaeriaceae: Genera and species known from culture. Stud. Mycol. 76:51-167.
Phipps, P. M., and Porter, D. M. 1998. Collar rot of peanut caused by Lasiodiplodia theobromae. Plant Dis. 82:1205-1209.

Ren, Y.-F., Bao, X.-T., Li, D.-X., Wang, Y., Wang, D.-L., Song, B.-A., and Chen, Z. 2019. Identification of the pathogen Lasiodiplodia theobromae causing tea leaf spot. Acta Phytopathol. Sin. 49:857-861 (in Chinese).

Robinson, M. D., McCarthy, D. J., and Smyth, G. K. 2010. Edger: A bioconductor package for differential expression analysis of digital gene expression data. Bioinformatics 26:139-140.

Rodríguez-Gálvez, E., Maldonado, E., and Alves, A. 2015. Identification and pathogenicity of Lasiodiplodia theobromae causing dieback of table grapes in Peru. Eur. J. Plant Pathol. 141:477-489.

Serrato-Diaz, L. M., Perez-Cuevas, M., Rivera-Vargas, L. I., and French-Monar, R. D. 2013. First report of Lasiodiplodia theobromae causing inflorescence blight of mango. Plant Dis. 97:1380.

Serrato-Diaz, L. M., Rivera-Vargas, L. I., Goenaga, R., and French-Monar, R. D. 2014. First report of Lasiodiplodia theobromae causing inflorescence blight and fruit rot of longan (Dimocarpus longan L.) in Puerto Rico. Plant Dis. 98: 279.

Woodward, J. E., Langston, D. B., Brock, J. H., Kemerait, C. J., Brenneman, T. B., and Beard, G. H. 2005. First demonstration of Koch's postulates for Lasiodiplodia theobromae fruit spot on eggplant (Solanum melongena). Plant Dis. 89: 687.

Yan, J.-Y., Xie, Y., Zhang, W., Wang, Y., Liu, J.-K., Hyde, K. D., Seem, R. C., Zhang, G.-Z., Wang, Z.-Y., Yao, S.-W., Bai, X.-J., Dissanayake, A. J., Peng, Y.$\mathrm{L}$., and Li, X.-H. 2013. Species of Botryosphaeriaceae involved in grapevine dieback in China. Fungal Divers. 61:221-236.

Yildiz, A., Benlioglu, K., and Benlioglu, H. S. 2014. First report of strawberry dieback caused by Lasiodiplodia theobromae. Plant Dis. 98:1579.

Zhang, X.-O., Dong, R., Zhang, Y., Zhang, J.-L., Luo, Z., Zhang, J., Chen, L.-L., and Yang, L. 2016. Diverse alternative back-splicing and alternative splicing landscape of circular RNAs. Genome Res. 26:1277-1287.

Zhang, X.-O., Wang, H.-B., Zhang, Y., Lu, X., Chen, L.-L., and Yang, L. 2014. Complementary sequence-mediated exon circularization. Cell 159:134-147.

Zheng, Q., Ozbudak, E., Liu, G., Hosmani, P. S., Saha, S., Flores-Gonzalez, M., Mueller, L. A., Rodrigues-Stuart, K., Dewdney, M. M., Lin, Y., Zhang, J., Tarazona, Y. C., Liu, B., Oliva, R., Ritenour, M. A., and Cano, L. M. 2021. Draft genome sequence resource of the citrus stem-end rot fungal pathogen Lasiodiplodia theobromae CITRA15. Phytopathology 111:761-764.

Zhu, H., Niu, X.-Q., Song, W.-W., Yu, F.-Y., Tang, Q.-H., Qin, W.-Q., and Chen, L.-Q. 2014. First report of leaf spot of tea oil camellia (Camellia oleifera) caused by Lasiodiplodia theobromae in China. Plant Dis. 98:1427. 